\title{
Crescimento e desenvolvimento motor de escolares de Muzambinho: um estudo com implicações acadêmicas, sociais e de política interinstitucional
}

\author{
Luciano Basso ${ }^{1}$ \\ Cássio Meira Júnior ${ }^{1}$ \\ Jorge Alberto Oliveira ${ }^{1}$ \\ Cláudia Lúcia de Moraes Forjaz ${ }^{2}$ \\ Januária Andréa de Souza ${ }^{3}$ \\ Antonio Prista ${ }^{4}$ \\ José António Ribeiro Maia 5 \\ Go Tani $^{1}$
}

https://doi.org/10.5628/rpcd.09.02-03.247

\section{RESUMO}

Este texto apresenta uma visão geral do estudo longitudinal misto acerca do crescimento físico e desenvolvimento motor de crianças e jovens e famílias Muzambienses. Instituições do Brasil, Portugal e Moçambique se associam para investigar o processo de crescimento e desenvolvimento de forma dinâmica e multifocal. Pretende interpretar as múltiplas interações que se estabelecem entre as variáveis e mapear as muitas facetas da mudança intra-individual e das diferenças inter-individuais. As implicações e os desafios são evidenciados pela adoção de uma abordagem multidiciplinar e multicentro em países de língua portuguesa. O estudo é operacionalizado em duas fases, com coletas semestrais: a primeira constou de 470 escolares dos 7 aos 12 anos de idade divididos em 4 coortes; e a segunda com 634 escolares dos 6 aos 14 anos de idade divididos em 5 coortes (ligadas à estrutura anterior), além da inclusão de 200 famílias nucleares dos escolares. São apresentados também alguns dos temas de investigação para cada uma das fases e as implicações académicas, sociais e inter-institucionais.

Palavras-chave: longitudinal, crescimento, desenvolvimento motor, famílias núcleares, coordenação motora

\begin{abstract}
${ }^{1}$ Laboratório de Comportamento Motor, Escola de Educação Física e Esporte, Universidade de São Paulo, Brasil

${ }^{2}$ Laboratório de Hemodinâmica da Atividade Motora, Escola de Educação Física e Esporte, Universidade de São Paulo, Brasil ${ }^{3}$ Escola Superior de Educação Física de Muzambinho e Fundação Educacional Muzambinho, Muzambinho-MG, Brasil ${ }^{4}$ Faculdade de Ciências de Educação Física e Desporto, Universidade Pedagógica de Moçambique, Maputo, Moçambique ${ }^{5}$ Laboratório de Cineantropometria e Gabinete de Estatística Aplicada, Faculdade de Desporto, Universidade do Porto, Portugal
\end{abstract}

\section{ABSTRACT \\ Growth and motor development of Muzambinho children and adolescents: a research project with academic, social and of inter-institutional political implications}

This paper presents a broad overview of a mixed-longitudinal study about growth and motor development of children, adolescents and their families from Muzambinho, Brazil. This study is multicenter, linking researchers from Brazil, Mozambique and Portugal aiming at the multifocal investigation about the dynamics of growth and development. It aims also at the interpretation of multiple interactions of a broad array of variables, as well as the varied facets of intraindividual change and interindividual differences. The implications and challenges are highlighted using a multidisciplinary and multicenter approach in Portuguese speaking countries. The study is devised in two phases with semester data collections: the first part sampled 470 subjects from 7 to 12 years divided in 4 cohorts; the second phase included 634 subjects from 6 to 14 years divided in 5 cohorts (linked to the structure of the first phase). We included also 200 nuclear families. We present some of the main research themes for each phase, their academic, social and inter-institutional implications.

Key-words: longitudinal, growth, motor development, nuclear families, motor coordination 


\section{INTRODUÇÃO}

Pesquisa de natureza descritiva e interpretativa acerca do modo como crianças e jovens crescem fisicamente, desenvolvem-se no domínio motor e social, relacionam-se com o seu corpo, percebem as suas potencialidades e sua saúde estão na ordem do inevitável. São de extrema relevância em termos pedagógicos, educativos e de saúde pública. Esta emergência informativa pauta-se, por exemplo, na necessidade de se obter um quadro detalhado e interativo de informações para que se possa caracterizar, compreender e estabelecer necessidades e prioridades de crianças e jovens que permitam definir linhas de ação no campo pedagógico do movimento humano(1, 2), tornando-o assim mais adequado(3) e culturalmente relevante. É também inequívoca a necessidade de identificar variáveis e situações que aumentem ou diminuam o risco das crianças se tornarem sedentárias quando adolescentes e/ou adultas ${ }^{(4)}$, para além do diagnóstico de prevalência de outras co-mobilidades de que se destacam o sobrepeso/obesidade e a emergência da síndrome metabólica. Essa busca é motivada pela forte constatação epidemiológica que tem apontado a prática de atividades físico-deportivas moderadas a vigorosas como benéfica para a saúde das crianças e jovens com potencial elevado para reduzir a incidência de fatores de risco das doenças crônico-degenerativas(5).

Esta agenda atual que associa pesquisadores de diferentes áreas da educação física, esporte e da saúde tem forte visibilidade em projetos europeus e norteamericanos de larga escala centrados em perspectivas multivariadas e interdisciplinares de alguns anos atrás e que ainda hoje estão extremamente ativos. Por exemplo, o estudo longitudinal de Lovaina (Leuven Longitudinal Study on Livestyle and Hearth) na Bélgica, acompanhou garotos dos 12 aos 18 anos de idade e depois dos 30 aos 40 anos. Inicialmente a preocupação central foi investigar o comportamento longitudinal do desenvolvimento da aptidão física, da maturação biológica, das características somáticas, e um conjunto bem diversificado de fatores de natureza fisiológica e sócio-culturais( ${ }^{(6)}$. Atualmente a sua perspectiva combina, aos aspectos anteriores, fatores clínicos, epidemiológicos e genéticos além de incluir toda a família do sujeito, pesquisando aspectos dos filhos quando os pais tinham as mesmas idades dos filhos (7). Outro exemplo é o projeto longitudinal misto de Amesterdam (Amsterdam Growth and Health Study), que analisou o desenvolvimento e o efeito de mais de 50 características de jovens no âmbito da antropometria, psicologia, hábitos nutricionais, atividade física, aptidão física e estilo de vida sobre a capacidade cardiorespiratória e nos fatores de risco de doenças cardiovasculares na idade adulta(8). Numa perspectiva de pesquisa longitudinal centrada nas famílias, o estudo de Fels (em Village Yellow Springs, EUA) pode ser apresentado como uma referência inequívoca. Com mais de 70 anos de história, agregando até 4 gerações de pessoas, tem diversos desdobramentos a partir da análise das características de estatura, peso, maturação, composição corporal, fatores de risco de doenças crónico-degenerativas, marcadores genéticos, atividade física, personalidade, inteligência, movimentos fetais e hábitos nutricionais ${ }^{(9)}$.

Um dos pontos centrais nos estudos relatados acima é a combinação de uma abordagem longitudinal fortemente interdisciplinar numa perspectiva multifacetada em termos de preocupações, enquadramento metodológico e elevado conjunto de variáveis que não se encontra frequentemente em estudos brasileiros sobre temas similares. A informação existente no Brasil é oriunda de pesquisas essencialmente transversais ou então longitudinais univariadas. Por exemplo, dentre pesquisas transversais, a das crianças e jovens de Santo André-SP(10), no final dos anos 60 do século passado, é ainda hoje utilizada como referência quando se discute o crescimento de crianças e jovens no Brasil(11). Um outro exemplo bem ilustrativo de pesquisas dessa natureza é a sequência de trabalhos de Guedes e Guedes ${ }^{(12-14)}$ que em termos gerais descrevem o status do desempenho motor e crescimento físico de crianças de 7 aos 17 anos de idade. Os únicos exemplos longitudinais localizados são o das crianças e jovens de Santa Catarina $(15,16)$ e do projeto PROESB-Brasil(17-19). No primeiro foi descrito o desenvolvimento das características antropométricas de forma transversal de 875 crianças e jovens de 7 aos 17 anos, 35\% das quais foram acompanhadas longitudinalmente por quatro anos(15). Já no segundo, Nahas e colaboradores ${ }^{(16)}$, investigaram a aptidão física relacionada a saúde de 84 crianças e jovens de 10 a 14 anos. Os estudos a partir do 
PROESB-Brasil buscaram identificar o desenvolvimento do índice de massa corporal(19), aptidão físi$\mathrm{ca}^{(18)}$ e do crescimento físico(17) de 70 crianças e jovens de 10 aos 14 anos de idade da cidade de Canoas/RS. Não obstante a relevância da informação produzida, é importante realçar a natureza descritiva dessas pesquisas, dado que se referem aos aspectos do status das crianças por faixa etária e gênero. A preocupação em obter informação relevante sobre o crescimento e desenvolvimento motor das crianças e jovens brasileiros tem uma história de cerca de 50 anos. No entanto, as suas limitações ficam claras quando se pretende investigar o processo de crescimento e desenvolvimento de forma dinâmica e multifocal, procurando interpretar as múltiplas interações que se estabelecem entre variáveis a que se associa a urgência em mapear as muitas facetas da mudança intra-individual e das diferenças inter-individuais. Essa visão pode ser gerada a partir dos elementos nucleares sobre o desenvolvimento, apresentadas por Baltes \& Nesselroade(20) e Burr \& Nesselroade(21) e dos trabalhos publicados num exemplar especial da International Journal of Sports Medicine(22), que se dedicou à apresentação de vários desafios desse tipo de investigação na área clínica, pedagógica e epidemiológica, além de sublinhar o seu impacto em termos de saúde pública.

O presente documento pretende apresentar aspectos da relevância investigativa, educativa e de relacionamento inter-institucional de um projeto de pesquisa por trazer à luz do dia a emergência em iniciar uma abordagem multidiciplinar e multicentro (internacional) acerca do crescimento e desenvolvimento motor de crianças e suas famílias. A estratégia que se utiliza na sua apresentação é a seguinte: em primeiro lugar tratar-se-á, genericamente, de alguns dos desafios que são colocados nas pesquisas desta natureza; em segundo lugar serão apresentadas, em separado, as idéias fulcrais do projeto, os seus objetivos e metodologias; em terceiro lugar são referidos alguns dos temas de investigação.

\section{DESAFIOS}

\section{Abordagem longitudinal mista}

É mais que evidente a riqueza descritiva e interpretativa oriunda de informação longitudinal, considerada um relato da história natural do desenvolvimento de múltiplas características e atributos dos sujeitos. Contudo, qualquer pesquisa longitudinal pura é morosa, de alto custo e com problemas sérios de drop-out. Uma alternativa altamente flexível, extremamente promissora e usada em diferentes pesquisas de elevado impacto educativo e epidemiológico é a que se refere ao delineamento longitudinal misto que contém os pontos fortes da pesquisa longitudinal e transversal (23). A este delineamento associa-se a possibilidade de acrescentar preditores de mudanças inter-individuais que também mudam no tempo de forma paralela e/ou defasada, bem como adicionar outros preditores fixos do sujeito em estudo. A versatilidade analítica e interpretativa com base na modelação hierárquica é elevada e as questões que podem ser respondidas com base nesta metodologia e delineamento não têm sido pensadas e realizadas no exato domínio de projetos sobre o crescimento, desenvolvimento motor e aptidão física da criança e jovens no espaço da lusofonia [sobre esse assunto ver (24)].

\section{Métodos multivariados}

Além de se recorrer a um delineamento longitudinal misto, é também necessário que a investigação explore, simultaneamente, múltiplas variáveis potenciais que influenciem o crescimento, o desenvolvimento motor e a aptidão física das crianças e jovens. Por exemplo, a identificação da relevância de preditores (invariantes ou não no tempo) que expliquem a variação inter-individual de uma determinada característica não deveria ser realizada exclusivamente a partir de análises univariadas, uma vez que distintas variáveis apresentam padrões distintos de inter-correlações significativamente diferentes de zero que reclamam o uso de métodos multivariados, de modo a permitirem uma interpretação mais abrangente e esclarecedora dos resultados disponíveis.

\section{Diferenciação dos níveis da informação}

De forma geral, as grandes pesquisas internacionais sobre o crescimento e desenvolvimento motor de crianças e jovens recorrem a abordagens multivariadas e longitudinais [ver a esse respeito (22)]. No entanto, recentes trabalhos $(25,26)$ têm apresentado a relevância da estrutura diferencial da informação em planos hierárquicos. Deve-se ter sempre presente a estrutura organizacional da informação (uma informação 
estruturada em planos hierárquicos que interagem entre si). Por exemplo, as crianças estão agrupadas em diferentes classes de aula, as classes em escolas diferenciadas, as escolas em áreas geográficas distintas, ou seja, existe uma hierarquia inequívoca na rede informacional. A ausência de consideração desta estrutura hierárquica, que salienta uma interligação ou dependência forte da unidade de análise mais baixa na hierarquia (alunos) da mais elevada (que podem ser classes, escolas, áreas geográficas, etc.,), acarreta uma leitura demasiadamente parcial e truncada da informação disponível. Daí a sugestão em atribuir maior importância a um pensamento e a uma estrutura analítica centrada na Modelação Hierárquica ou Multinível. Vale ressaltar que estudos longitudinais também apresentam uma estrutura multinível, sendo o primeiro nível constituído por informações repetidas no tempo e o segundo pelos sujeitos e suas características. Convém ter sempre presente que um delineamento hierárquico implica um processo amostral situado nos diferentes planos da estrutura conceitual dos problemas. Assim, o desafio se coloca na urgência em estabeler um quadro teórico e metodológico no âmbito da modelação hierárquica ou multinível da informação para a elaboração de projetos sobre o crescimento, desenvolvimento motor e aptidão física da criança e jovens.

\section{Diferentes implicações: educação e saúde}

É mais que evidente que estudos longitudinais mistos e multivariados sobre crescimento e desenvolvimento motor são altamente desafiadores pelos distintos desdobramentos explícitos no plano dos problemas, assim como pela utilidade social do uso da informação. A idéia central é um desafio à interdisciplinaridade na literatura científica e social dos resultados da pesquisa. Um empreendimento longitudinal consome muito tempo e trabalho para superar inúmeros desafios metodológicos. Desse modo a sua estruturação deva ser planejada agregando pesquisadores de várias áreas do conhecimento em finalidades diferenciadas. Uma integração atual é a que agrega áreas de influência da educação e saúde. Decorre daqui a sua contribuição para a ação do professor, na busca de entender melhor os diversos aspectos do processo de desenvolvimento das crianças e assim elaborar novas formas de intervenção; também é possível gerar informações facilmente interpretáveis para os pais e responsáveis no acompanhamento e controle de eventuais estados de risco para a saúde da criança e dos jovens.

\section{Políticas interinstitucionais}

Fazer ciência nos dias de hoje implica uma rede de investigadores de diferentes instituições com interesse em temas comuns de pesquisa. Do ponto de vista das instituições, portanto, viabilizar o bom funcionamento dessa rede constitui-se uma preocupação central da sua política de relações interinstitucionais. As relações entre instituições podem ser estabelecidas em diferentes frentes: intercâmbio de estudantes e de docentes, programas de co-tutela tanto na graduação como na pós-graduação, dulpo diploma, somente para citar as mais comuns. No entanto, uma relação produtiva e duradoura se estabelece de fato quando o inter-relacionamento ocorre no âmbito da investigação em torno de projetos de pesquisa de qualidade devidamente avaliada por órgãos competentes e com o envolvimento de laboratórios de pesquisa constituídos.

\section{PROJETO: OBJETIVOS, MATERIAIS E MÉTODOS 3.1. Primeira fase Objetivos gerais}

A primeira fase do projeto intitulada

"Desenvolvimento motor de crianças brasileiras e portuguesas: estudo longitudinal misto do desenvolvimento de aspectos da coordenação motora e da aptidão física" teve como essência investigar tanto aspectos diversificados do crescimento somático e desempenho motor quanto à associação entre a coordenação motora e à aptidão física, permitindo vislumbrar as possíveis influências que esses dois sistemas exercem entre si.

\section{Objetivos específicos}

I. Investigar os padrões de estabilidade e mudança na coordenação motora, aptidão física associada à saúde e atividade física.

II. Pesquisar os valores de sobrepeso e obesidade, bem como a sua influência na coordenação motora e atividade física.

III. Identificar aspectos relacionados com os níveis de prontidão coordenativa e da aptidão física.

IV. Associar níveis distintos de sedentarismo e eleva- 
da atividade física aos desempenhos coordenativos e da aptidão física.

V. Investigar as influências do ambiente (estatuto sócio-econômico da família, espaço, qualidade e quantidade de material disponível nas escolas) nos resultados da coordenação motora e aptidão física associada à saúde.

VI. Identificar crianças com problemas de obesidade, insuficiência coordenativa e de aptidão e encaminhálas para programas escolares de enriquecimento motor.

VII. Promover metodologias de pesquisa e análise de dados no domínio do crescimento e desenvolvimento motor aos pesquisadores do espaço da língua portuguesa.

\section{Amostra}

Participaram voluntariamente do estudo 471 escolares muzambinhenses-MG, na faixa de 7 aos 12 anos de idade. A cidade de Muzambinho, localizada na região sudoeste do Estado de Minas Gerais, foi escolhida por oferecer condições logísticas, técnicas e administrativas para o desenvolvimento do projetoii. Uma parceria foi definida com a Escola Superior de Educação Física de Muzambinho (ESEFM) e com a Secretaria Municipal de Educação da cidade. A amostra foi composta por $51 \%$ de meninos e $49 \%$ de meninas e a participação foi condicionada à autorização dos pais ou responsáveis, que assinaram termos de consentimento livre e esclarecido. O projeto foi aprovado pelo Comitê de Ética em Pesquisa da EEFEUSP e recebeu o registro 13832 no Conselho Nacional de Ética em Pesquisa (CONEP - Ministério da Saúde).

\section{Delineamento}

Essa fase do projeto teve a duração de dois anos e meio, sendo que as crianças foram avaliadas a cada seis meses. As crianças foram divididas em quatro coortes de acordo com a faixa etária apresentada na Tabela 1.

Foi imprescindível a formação e o treinamento de uma equipe de trabalho. A equipe principal de coleta foi constituída por 25 integrantes (dentre os quais três docentes) do Laboratório de Comportamento Motor da Escola de Educação Física e Esporte da Universidade de São Paulo e um docente da
Tabela 1. Distribuição das idades por coortes ao longo das cinco coletas.

\begin{tabular}{llllllllllll}
\hline Coorte & \multicolumn{1}{c}{} \\
\hline 1 & 7 & 7,5 & 8 & 8,5 & 9 & & & & & \\
\hline 2 & & 8 & 8,5 & 9 & 9,5 & 10 & & & \\
\hline 3 & & & & 9 & 9,5 & 10 & 10,5 & 11 & \\
\hline 4 & & & & & & & 10 & 10,5 & 11 & 11,5 & 12 \\
\hline
\end{tabular}

Universidade Pedagógica de Moçambique. Juntaramse a esse grupo, ainda, três docentes da Escola Superior de Educação Física de Muzambinho. A fase da preparação da equipe constou de uma ação de formação teórico-prática para familiarizar e treinar a equipe com os protocolos de avaliação.

Posteriormente, com o objetivo de identificar e sanar eventuais problemas associados à execução dos testes, foi realizado um estudo piloto na Escola de Educação Física e Esporte da Universidade de São Paulo com crianças de 9 e 10 anos. As coletas foram realizadas nas dependências da Escola Superior de Educação Física de Muzambinho, dois ginásios poliesportivos e uma pista de atletismo. Também foi recrutada uma equipe auxiliar, composta de 20 alunos de graduação de educação física desta mesma escola.

\section{Variáveis de estudo}

As variáveis selecionadas foram consideradas suficientes para mapear diferentes aspectos do crescimento físico, da aptidão física, do domínio motor (capacidades e habilidades motoras), da atividade física, da postura das crianças e nível socioeconômico (Tabela 2).

\section{Controle da qualidade da informação coletada}

Todos os dados recolhidos no momento da coleta foram passados para uma ficha de anotações de cada criança e depois digitados em dupla entrada em bases de dados distintas geradas no Acess MicrosoftOffice. Após a digitação, os dados das duas bases foram comparados e quando ocorria discordância, voltava-se à ficha da criança para encontrar a entrada correta. A exceção foi para os dados do TGMD-II, pois a avaliação das habilidades motoras ocorreu via 
Tabela 2. Dimensão, variáveis e referências utilizadas na realização da fase 1.

\begin{tabular}{lll}
\hline Dimensão & Variáveis & Referências \\
\hline Crescimento físico & Altura e Peso & International Working \\
& Goroup on Kinanthropometry (27) \\
\hline Aptidão física & Flexibilidade & Boileau et al. (28) \\
& Resistência muscular & Fitnessgram (29) \\
& Força muscular & \\
\hline Domínio motor & Resistência cardiorrespiratória & \\
\cline { 2 - 3 } & Habilidades motoras básicas & TGMD-II (30) \\
\cline { 2 - 3 } & Capacidades motoras: coordenação motora grossa, & KTK (31) \\
& timing coincidente, tempo de reação simples e & Pease (32), Santos e colab. (33), \\
\hline Atividade física & de movimento & Freudenheim e Tani (34) \\
\hline Postura & Nível de atividade física & Godin e Shephard (35) \\
\hline Sócio-Econômica & Curvaturas e angulações articulares & Palmer e Epler (36) \\
\hline
\end{tabular}

análise de vídeo realizada posteriormente à coleta. Cada habilidade motora dessa bateria foi analisada por um avaliador, que iniciou o trabalho após apresentar níveis de concordância intra e inter-avaliador acima de $85 \%(30)$.

\section{Temas de estudo}

Os temas de estudo apresentados aqui possibilitam, em termos da sua representatividade, apreender uma amostra da visão geral dos tipos de análises que foram planejadas a partir da execução da primeira fase do projeto.

\section{Temas:}

1. Atividade física, aptidão física e crescimento somático.

2. Dificuldades no domínio das habilidades motoras básicas.

3. Diversidade na coordenação motora e preditores de mudança.

4. Mudança e estabilidade das capacidades motoras (timing antecipatório, tempo de movimento e tempo de reação).

5. Relações temporais entre capacidades motoras e habilidades motoras.

6. Epidemiologia dos problemas posturais.

7. Avaliação transcultural das baterias KTK e TGMD-II: Uma análise centrada em modelos de estrutura de covariância. 8. Diagnóstico de crianças com dificuldades motoras: a relação KTK e TGMD-II.

9. Analise do crescimento e desenvolvimento motor de crianças e jovens no Brasil, Portugal e Moçambique.

\subsection{Segunda fase \\ Objetivo geral}

Além de continuar a investigação dos objetivos da primeira fase do projeto (extensivo a mais dois anos de pesquisa), a segunda fase iii intitulada "Crescimento, desenvolvimento motor e saúde de crianças, adolescentes $e$ famílias nucleares: um estudo dos fatores de risco e dos efeitos genéticos, ambientais e suas interações" tem a novidade de centrar-se na pesquisa da agregação familiar das capacidades motoras, físico-motoras e dos fatores de risco cardiovascular.

\section{Objetivos específicos}

I. Avaliar o risco cardiovascular de crianças e seus pais, estabelecendo a relação com a aptidão aeróbia e prática de atividades físicas.

II. Avaliar o grau de agregação familiar dos fatores de risco, da aptidão aeróbia e da prática de atividade física. 
Tabela 3. Distribuição das idades por coortes ao longo das nove coletas. As células em preto representam as idades já coletadas na primeira e segunda fase para cada coorte ${ }^{4}$

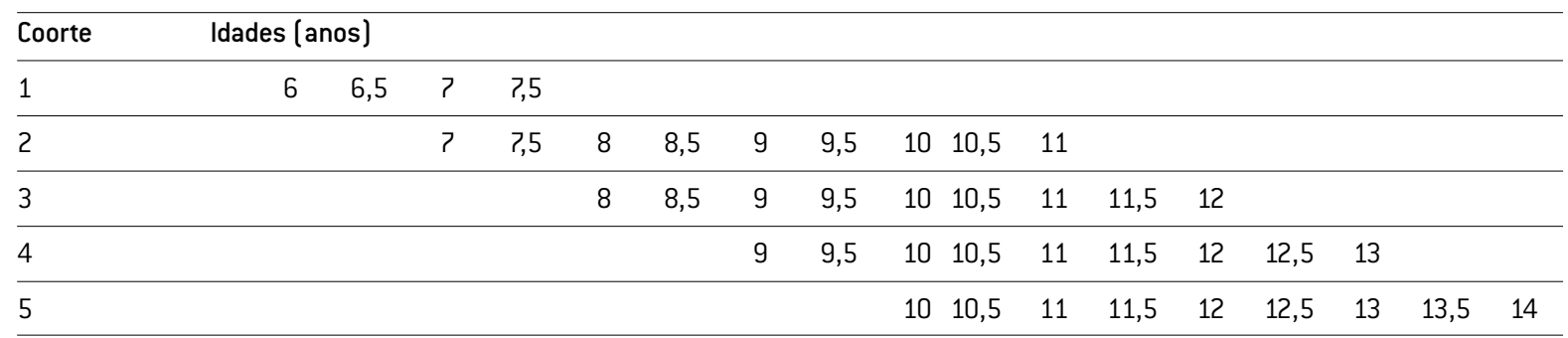

Tabela 4. Dimensão, variáveis e protocolos das duas fases do projeto (em negrito e itálico estão presentes somente na segunda fase).

\section{$1^{\text {a }}$ FASE}

\begin{tabular}{|c|c|c|}
\hline Dimensão & Variáveis & Referências \\
\hline \multirow[t]{3}{*}{ Crescimento físico } & Altura & International Working \\
\hline & Peso & Group on Kinanthropometry (27) \\
\hline & Porcentagem Gordura corporal & Boileau et al. (28) \\
\hline \multirow[t]{4}{*}{ Aptidão física } & Flexibilidade & Fitnessgram (29] \\
\hline & Resistência muscular & \\
\hline & Força muscular & \\
\hline & Resistência cardiorrespiratória & \\
\hline \multirow[t]{4}{*}{ Domínio motor } & Habilidades motoras básicas & TGMD-\|l (30) \\
\hline & Capacidades motoras: coordenação motora grossa, & KTK (31) \\
\hline & timing coincidente, tempo de reação simples e & Pease (32), Santos et al. (33), \\
\hline & de movimento & Freudenheim e Tani (34) \\
\hline Atividade física & Nível de atividade física & Godin e Shephard (35) \\
\hline Postura & Curvaturas e angulações articulares & Palmer e Epler (36) \\
\hline Sócio-Econômica & Critério de Classificação Econômica Brasil & IBGE (37) \\
\hline
\end{tabular}

\section{$2^{\mathrm{a}}$ FASE}

\begin{tabular}{|c|c|c|}
\hline Psicológico & Traços da personalidade & ETPC (38) \\
\hline Atividade física & Nível e padrão de atividade física & Acelerometria (39) \\
\hline \multirow[t]{2}{*}{ Domínio motor } & Habilidades motoras & Haywood (40) \\
\hline & Capacidades motoras: preensão manual & Ugrinowitsch, et. al. (41) \\
\hline \multirow[t]{4}{*}{ Risco cardiovascular dos pais } & Sintomas e doenças & ACSM (42) \\
\hline & Uso de Remédios & \\
\hline & Fatores de risco cardiovasculares & \\
\hline & Medidas metabólicas & \\
\hline Aptidão física dos pais & Capacidade aeróbia & Astrand [42] \\
\hline \multirow[t]{2}{*}{ Risco cardiovascular da criança } & Sintomas e doenças & $\operatorname{ACSM}(42)$ \\
\hline & Fatores de risco & \\
\hline
\end{tabular}


III. Avaliar o produto e o processo de desenvolvimento das habilidades motoras ao longo das idades. IV. Identificar crianças propensas ao desenvolvimento de fatores de risco (obesidade, insuficiência coordenativa e aptidão física) e propôr programas escolares de enriquecimento motor.

\section{Amostra}

A estimativa final de estudo foi de $25 \%$ da população escolar de Muzambinho/MG. A amostra final das duas fases é subdivida por 634 crianças na faixa etária de 06 a 14 anos de idade - 471 referentes a primeira fase com idades entre 7 a 14 anos e de 163 crianças da segunda fase com idades entre seis e dez anos. A amostra referente às famílias será composta de aproximadamente 200 famílias nucleares.

\section{Delineamento}

Essa fase do projeto tem a duração de dois anos, sendo que as crianças serão avaliadas a cada seis meses. As crianças foram divididas em cinco coortes de acordo com a faixa etária (Tabela 3).

A coleta de dados das famílias nucleares será realizada entre os quatro momentos de avaliação.

\section{Variáveis de estudo}

Além das variáveis do primeiro projeto também foram adicionadas outras que evidênciam os diferentes aspectos da dimensão da atividade física, risco cardiovascular, aptidão física, domínio motor e psicológico conforme descrito na Tabela 4. As únicas exceções são: a) a atividade física que também será descrita por acelerômetros e b) a habilidade motora que também será avaliada sob o aspecto do produto da ação. Vale ressaltar que para as famílias nucleares foram apenas coletadas informações sobre o risco cardiovascular, capacidades aeróbia e motoras.

\section{Controle da qualidade da informação}

O controle da informação será realizado tal como na fase 1 .

\section{Temas de estudo e resultados esperados}

Os temas de estudo apresentados aqui possibilitam, em termos da sua representatividade, uma visão geral dos tipos de análises e temas de investigação que foram planejados para a segunda fase do projeto.

\section{Temas:}

1. Relação entre processo e produto da ação motora ao longo da infância e juventude.

2. Agregação familiar dos fatores de risco cardiovascular.

3. Modelação hierárquica do desempenho motor e seus preditores.

4. Relação entre factores de risco cardiovascular, niveis de atividade física e aptidão física.

5. Relação entre prevalência de sobrepeso, obesidade, níveis de sedentarismo e fatores de risco cardiovascular.

6. Crianças e jovens em risco ponderal e metabólico e a sua associação com a insuficiência de desempenho motor.

7. Modelação hierárquica da coordenação motora e seus possiveis preditores.

8. Construção das cartas centílicas do crescimento e diferentes aspectos do desenvolvimento motor. Utilidade educativa e pedagógica.

9. Agregação familiar das capacidades perceptivo-motoras e físico-motoras.

10. Estudo exploratório acerca da noção de prontidão motora.

\section{CONSIDERAÇÕES FINAIS}

A abordagem longitudinal-mista do projeto em apreço, interdiciplinar na sua concepção, multivariada na estrutura relacional das suas variáveis e centrada também nas famílias acerca de diferentes facetas do seu crescimento, desenvolvimento e saúde possibilita uma quantidade enorme de informação com um nível elevado de complexidade para a qual urge a necessidade de dar ordem e atribuir significado acadêmico e social.

Do ponto de vista acadêmico irá desaguar em diferentes produtos metodólogicos e substantivos acerca do valor do delineamento da pesquisa, dos procedimentos de análise e do esclarecimento de aspectos relacionais entre crescimento físico, desempenho e desenvolvimento motor. A leitura interdisciplinar dos fenômenos em apreço trará novidade sob a forma de explorações nos domínios teórico e metodológico, de novos questionamentos e desafios de pensamento e ação num território em que se pretende ter um maior número de investigadores lusófonos.

Na perspectiva da atuação pedagógica será possível fornecer aos professores uma visão mais precisa e atualizada das múltiplas características dos seus alunos ao longo de toda a segunda infância e adolescên- 
cia. Diante dessa visão será possível elaborar propostas didáticas mais sustentáveis com a diversidade de estados de prontidão dos alunos. O mapeamento dos momentos mais oportunos para a intervenção é um aspecto de grande impacto na melhoria do processo ensino-aprendizagem.

A relevância social deste empreendimento é inquestionável. Em primeiro lugar pela gratuitidade da avaliação e serviço prestado às crianças e às suas famílias. Em segundo lugar pelo valor de "saúde pública preventiva" adstrito ao diagnóstico implícito neste projeto. Em terceiro lugar, pelo caráter interativo de preocupações e proposta de solução da Prefeitura bem como da Escola Superior de Educação Física de Muzambinho e Escola de Educação Física e Esporte da USP a que se associam instituições de Portugal e Moçambique em prol do crescimento saudável e desenvolvimento harmonioso sustentado de crianças e jovens a que se combina uma preocupação com a saúde da sua família.

\section{COMITÊ DE ÉTICA}

Conselho Nacional de Ética em Pesquisa (CONEP Ministério da Saúde) processo número 13832.

\section{FINANCIAMENTO}

Projeto financiado em Edital Universal do CNPq 19\2004 - Processo 478202/2004-0.

Projeto financiado em Edital MCT/CNPq 15/2007 Processo 478249/2007-1.

Bolsa de Estágio de doutoramento no Exterior pela

Capes - Processo no 4434/07-2

\section{AGRADECIMENTOS}

Os autores agradecem ao CNPq pelo financiamento das duas fases do projeto, aos professores Willian Peres Lemos, Elisângela Silva, Wagner Zeferino, aos alunos de graduação da Escola Superior da Educação Física de Muzambinho e da graduação e pós-graduação da Escola de Educação Física e Esporte da USP que participaram da organização e coleta de dados.
NOTAS

${ }_{1}^{1}$ Projeto financiado em Edital MCT/CNPq 15/2007 - Processo 478249/2007-1.

${ }^{2} E$ importante ressaltar que a escolha desta cidade não se pautou em nenhum critério metodológico de amostragem; não se assume que seja representativa do estado de Minas Gerais, muito menos do Brasil.

${ }^{3}$ Projeto financiado em Edital Universal do CNPq 19\2004 - Processo 478202/2004-0.

${ }^{4}$ Coletas realizadas até Novembro de 2008 (data de submissão deste artigo).

\section{CORRESPONDÊNCIA}

Go Tani

Departamento de Pedagogia do Movimento do Corpo Humano

Escola de Educação Física e Esporte - USP

Av. Prof. Mello Moraes, 65 - Cidade Universitária -

São Paulo, São Paulo

CEP 05508-030

E-mail: gotani@usp.br 


\section{REFERÊNCIAS BIBLIOGRÁFICAS}

1. Tani G (1987). Educação física na pré-escola e nas quatro primeiras séries do primeiro grau: uma abordagem desenvolvimentista I. Revista Kinesis, 3:19-41.

2. Tani G (2001). Educação física na educação infantil: pesquisa e produção do conhecimento. Rev Paulista de Educação Física, supl. 1:110-115

3. Halverson LE (1966). Development of motor patterns in young children. Quest, 6: 44-53

4. Dishman RK, Washburn RA, Heath GW (2004). Physical activity epidemiology. Human Kinetics

5. Strong WB, Malina RM, Blimkie CJR, Daniels SR, Dishman RK, Gutin B, et al. (2005). Evidence based physical activity for school-age youth. J Pediatr, 146(6): 732-737.

6. Beunen G, Ostyn M, Simons J, Renson R, Claessens AL, Vanden Eynde B, et al (1997). Development and tracking in fitness components: Leuven longtudinal study on lifestyle, fitness and health. Int J Sports Med, 18 (Suppl 3): S171S178.

7. Matton L (2006). Secular trend, tracking and familial tresemblance in physical activity and physical fitness: The Leuven longitudinal study on lifestyle, fitness and health 1969-2004. Leuven: Katholieke Universiteit Leuven

8. Kemper HC, van Mechelen W, Post GB, Snel J, Twisk JW, van Lenthe FJ, et al (1997). The Amsterdam Growth and Health Longitudinal Study. The past (1976-1996) and future (1997-?). Int J Sports Med, 18 (Suppl 3): S140- S150

9. Roche AF, Guo SS, Towne B (1997). Opportunities and difficulties in long-term studies of growth. Int J Sports Med, 18 (Suppl 3): S151-61

10. Marcondes E (1982). Normas para o diagnóstico e a classificação dos distúrbios do crescimento e da nutrição - última versão. Pediatria, 4: 307-26

11. Glaner MF (2003). Referenciais para o crescimento físico de adolescentes gaúchos e catarinenses. Rev Bras Cineantropom Desempenho Humano, 5:17-26

12. Guedes DP, Guedes JERP (1993). Aptidão física relacionada à saúde de crianças e adolescentes: avaliação referenciada por critério. Rev Bras de Atividade Física e Saúde, 1(2): 27 38.

13. Guedes JERP, Guedes DP (1995). Características de crescimento em crianças e adolescentes do município de Londrina - Paraná. Rev Associação dos Professoras de Educação Física de Londrina, 10(17): 56-70

14. Guedes DP, Guedes JE (1993). Crescimento e Desempenho Motor em Escolares do Município de Londrina, Paraná, Brasil. Cad Saú Pública, 9(Suppl 1): 58-70

15. Waltrick ACA, Duarte MFS (2000). Estudo das caracteristicas antropométricas de escolares de 7 a 17 anos - uma abordagem longitudinal mista e transversal. Rev Bras Cineantropom Desempenho Humano, 2(1):17-30

16. Nahas MV, Petroski EL, Jesus JF, Silva OJ (1992). Crescimento e aptidão física relacionada à saúde em escolares de 7 a 10 anos - Um Estudo longitudinal. Rev Bras Ciência do Esporte, 14(1): 7-17.

17. Bergmann GG, Bergmann GG, Pinheiro ES, Moreira RB, Marques AC, Gaya A (2008). Estudo longitudinal do crescimento corporal de escolares de 10 a 14 anos: dimorfismo sexual e pico de velocidade. Rev Bras Cineantropom Desempenho Humano, 10(3): 249-54

18. Bergmann GG, Bergmann MLA, Moreira RB, Gaya A (2007). Desenvolvimento e estabilidade da aptidão muscu- lar em escolares de 10 a 14 anos. Ciência e Conhecimento Revista Eletrônica da ULBRA São Jerónimo, 1:1-10

19. Bergmann GG, Bergmann MLA, Moreira RB, Gaya A (2007). Desenvolvimento do índice de massa corporal: estudo longitudinal com escolares dos 10 aos 14 anos de Idade. Ciência e Conhecimento - Revista Eletrônica da ULBRA São Jerónimo, 2.

20. Baltes PB, Nesselroade JR (1979). History and rationale of longitudinal research. In: Nesselroade JR, Baltes PB, editors. Longitudinal research in the study of behavior and development. New York: Academic Press, p. 1-39.

21. Burr JA, Nesselroade JR (1990). Change measurement. In: von Eye A, (Editor), Statistical methods in longitudinal research: principles and structuring change., San Diego: Academic Press, p. 3-34.

22. van Mechelen W, Mellenbergh GJ (1997). Problems and Solutions in Longitudinal Research. Int J of Sport Medicine, 18(sup. 3): S139-S254.

23. Freitas DL, Maia J, Beunen G, Lefevre J, Calaessens A, Marques A, et al (2002). Crescimento somático, maturação biológica, aptidão física e estatuto sócio-económico de crianças e adolescentes madeirenses. Funchal: Universidade da Madeira

24. Maia J, Carneiro M, Silva RG, Pereira S, Seabra A, Bustamante A, et al. (2006). Um esforço de interpretação empírica da variação nos níveis de aptidão física a partir da modelação hierárquica: um estudo em crianças dos seis aos 10 anos de idade da região de Amarante - Portugal. Rev Bras de Educação Física e Esporte, 20(1): 71-82

25. Maia JA, Garganta R, Seabra A, Lopes VP, Vinagre J, Freitas DL, et al (2005). Dados longitudinais e modelação hierárquica: um tutorial para investigadores das ciências do desporto. Rev Bras Cineantropom Desempenho Humano, 7(2): 94108

26. Maia JA, Beunen G, Lefevre J, Claessens AL, Renson R, Vanreusel B (2003). Modeling stability and change in strength development: a study in adolescent boys. Am J Hum Biol, 15(4): 579-591

27. Ross W, Marfell-Jones M (1983). Kinanthropometry. In: Macdougall J, Wenger H, Green H, (Editors), Physiological Testing of the Elite Athlete. New York: Movement Publications, p. 75-115.

28. Boileau RA, Lohman TG, Slaughter MH (1985). Exercise and body composition of children and youth. Scand J Med Sci Sports, 7:17-27.

29. Meredith MD, Welk GJ (1999). Fitnessgram test administration manual. $2^{\text {nd }}$ ed. Champaign: Human Kinetics

30. Ulrich DA (2000). Test of gross motor development II. $2^{\text {nd }}$ ed. Austin, Texas: Pro-Ed

31. Schilling F, Kiphard EJ (1974). Körperkoordinationstest für kinder, KTK. Weinheim: Beltz Test Gmbh

32. Pease DG (2000). Motor behavior concepts: equipment selection and application for teaching and research. Lafayette, IN

33. Santos S, Tani G (1995). Tempo de reação e a aprendizagem de uma tarefa de timing antecipatório em idosos. Rev Paulista de Educação Física, 9(1): 51-62

34. Freudenheim AM, Tani G (1995). Efeitos da estrutura de prática variada na aprendizagem de uma tarefa de timing coincidente em crianças. Rev Paulista de Educação Física, 9(2): 87-98

35. Godin G, Shephard RJ (1985). A simple method to assess exercise behavior in the community. Can J Appl Sport Sci, 10(3): 141-146 
36. Palmer M, Epler M (2000). Fundamentos das Técnicas de Avaliação Musculoesquelética. Rio de Janeiro: Guanabara Koogan

37. IBGE (2004). Estimativas de população para o Brasil em 2004. Saúde/Datasus Md, editor. Brasília

38. Sisto FF (2004). Escala de Traços de Personalidade para Crianças. São Paulo: Vetor Editora Psicopedagógica Ltda

39. Oliveira MM, Maia JA (2001). Avaliação da actividade física em contextos epidemiológicos. Uma revisão da validade e fiabilidade do acelerómetro Tritrac-R3D, do pedómetro Yamax Digi-Walker e do questionário de Baecke. Rev Port Ciên Desporto, 1(3): 73-88.

40. Haywood KM (2005). Life span motor development. $4^{\text {th }}$ ed. Champaign: Human Kinetics

41. Ugrinowitsch H, Tertuliano IW, Coca AA, Pereira FAdS, Gimenez R (2003). Freqüência de feedback como um fator de incerteza no processo adaptativo em aprendizagem motora. Rev Bras Ciên Mov, 11(2): 41-7

42. American College of Sports Medicine (2006). Diretrizes do ACSM para os testes de esforço e sua prescrição.

Guanabara Koogan 